\title{
Conceptual Change Through Building Three-Dimensional Virtual Models
}

\author{
Michael Barnett ${ }^{1}$, Thomas Keating ${ }^{2}$, Sasha A. Barab ${ }^{1}$, Kenneth E. Hay ${ }^{3}$ \\ ${ }^{1}$ Indiana University, School of Education 201 N. Rose Ave. Bloomington, IN. 47405-1006 \\ ${ }^{2}$ Boston College, Campion Hall Room 123, Chestnut Hill, MA 02467-3813 \\ ${ }^{3}$ University of Georgia, 611 Aderhold. Athens, GA 30602 \\ Tel: (812) 856-8473, Fax: (812) 856-8239 \\ Email: gmbarnet@indiana.edu, keatingt@bc.edu, sbarab@indiana.edu, khay@coe.uga.edu
}

\begin{abstract}
In this study we assessed the growth of university students' conceptual understanding of the seasons of the Earth, and eclipses and phases of the Moon upon completing a threedimensional (3-D) modeling-based Introduction to Astronomy course. Interviews prior to the course identified a range of student alternative frameworks regarding the composition and mechanics of the Solar System. During the course, student groups constructed three 3-D models of increasing complexity representing different aspects of the Solar System. Students explored these models in a 3-D virtual environment and posted their final projects on the course World Wide Web page. Post interviews were targeted toward quantitative and qualitative assessment of student conceptual growth. Students showed significant improvement in their conceptual understanding concerning the phases and eclipses of the Moon and reasons for the seasons. Results also suggest that 3-D modeling can be used to support students' understanding of many fundamental astronomy topics.
\end{abstract}

Keywords: science education, modeling/models, virtual reality, visualization

\section{Introduction}

In this study we examined how undergraduate university students' conceptual understanding of astronomy changed as a result of participating in an experimental introductory astronomy course. Students used Virtual Reality (VR) modeling software to construct their own 3-D models of different astronomical phenomenon such as; the seasons of the Earth, phases of the Moon, lunar and solar eclipses, and the scale and mechanics of the entire Solar System. Students were encouraged to explore and pose questions with their models; the goal being that they would develop deep understandings of the same astronomy concepts featured in traditional astronomy lecture courses.

The Virtual Solar System Course (VSS) described in this paper is one of the first attempts to integrate 3-D virtual reality modeling as a central component of an introductory astronomy course (Barab, Hay, Squire, Barnett, Schmidt, Karrigan, Yamagata-Lynch, \& Johnson, in press; Hay, Johnson, Barab, \& Barnett, in press). It is important to clarify that the type of virtual reality used in this course was not immersive, but rather a "window on the world", in which the students used software that emulated a 3-D environment on their two-dimensional (2-D) computer screens (McLellan, 1996). In this paper, we first present our theoretical framework, followed by a description of the course, our methodology, results and we end with a discussion of our results and discussion of some implications of our results for astronomy education.

\section{Theoretical Framework}

Over the past decade there has been a proliferation of studies (116 studies since 1988 according to the Pfundt \& Duit [1998] bibliography) reporting students' difficulties in understanding basic astronomical phenomenon (Parker, \& Heywood, 1998; Treagust, \& Smith, 1989). For example, in the notable film A Private Universe (Pyramid Film \& Video, 1988), Harvard students, faculty, and alumni were found to hold incorrect conceptions concerning the causes of the Earth's seasons, and other astronomical phenomena. Further, Atwood and Atwood (1996) found that only one of 49 pre-service science teachers could provide a scientifically accurate explanation of the causes of the Earth's seasons. In addition Fraknoi (1996) reported that in 1988 the Public Opinion Laboratory at Northern Illinois University conducted a survey that revealed only $45 \%$ of United States adults could correctly state that the Earth orbited the Sun and that it took a year to complete the trip. These impoverished understandings have been referred to by a myriad of terms in the literature (e.g. alternative conceptions, misconceptions just to name 
two). In this paper we will refer to these views as alternative frameworks, because understanding of many astronomy concepts are frequently embedded within a larger structure (Smith, diSessa, \& Roschelle, 1993).

Several researchers have concluded that students' alternative frameworks are resistant to change and are impediments to obtaining the accepted scientific framework (Clement, Brown, \& Zietsman, 1989). They recommend that science instruction should be focused at the replacement of students' alternative frameworks in favor of more scientifically accurate conceptions (Gilbert and Watts, 1983). Contrasting this view is a growing body of research that claims this replacement view is inadequate because it implies a simple one-to-one process where an incorrect concept supplants a correct concept (Demastes, Good, \& Peebles, 1995; Strike \& Posner, 1992). Whereas, in practice, conceptual understanding is an evolutionary process that emerges from a complex interplay between prior understanding and the context in which learning occurs. In this evolutionary view, alternative frameworks are a starting position to the construction of robust scientific understandings. This process is facilitated when students are exposed to a curriculum that provides an experiential base in which students are afforded the opportunity to test and investigate their existing understanding through meaningful activities (Smith, diSessa, \& Roschelle, 1993).

Modeling activities are becoming more commonplace in science classrooms, in part because educators have recognized that model-based reasoning can serve as medium that facilitates the shift from personal, intuitive knowledge (e.g. student held alternative frameworks), to a more mathematical-scientific understanding of the natural world (Lehrer, Horvath, Schauble, 1994). When students are engaged in constructing their own models they develop a conceptual understanding of phenomena through an iterative process of stating their own conceptual understanding, then testing their understanding by exploring the consequences of changing the parameters of their model, and finally revising their model to better match the actual phenomenon under study (Confrey \& Doerr, 1994). The growing power of computers, coupled with a reduction in cost, has created the opportunity to use desktop 3-D virtual reality and computational modeling as inquiry approaches to learning and the teaching of science (Barab, et. al., in press; Stratford, Krajcik, \& Soloway, 1998). A computational model is the embodiment of a concrete concept or theory, and creates a structure through with students can explore and experiment. Therefore, students can "do science" rather than being taught about science, because they are using techniques and tools that are similar to those employed by many scientists to investigate phenomena (Stewart, Hafner, Johnson, \& Finkel, 1992). Perhaps the most important aspect that computational modeling adds to traditional scientific inquiry in the classroom is the ability for students to visualize abstract concepts (e.g. Sadler, Whitney, Shore, \& Deutsch, 1999).

Astronomy is a difficult subject to teach to students because it requires students to gain an abstract understanding of dynamic relationships and events that take place in 3-D space (Parker \& Heywood, 1998). Despite the 3-D nature of astronomy, many of the resources available to students are in the form of 2-D charts, pictures, textbooks and slides. The emphasis of the VSS course is to have students actively engage in the construction of 3-D models. This is a shift away from traditional learning astronomy activities in which students are required to read or study descriptions of 3-D concepts usually within a two 2-D format such as a textbook or computer screen and then visualize the 2-D image as a 3-D system (Parker \& Heywood, 1998). This can be a difficult task, that can become very frustrating and the students can easily lose interest, or worse develop an alternative framework (Copolo, \& Hounshell, 1995; Khoo \& Koh, 1998). However, until recently, being able to model in 3-D meant gaining access to and time on expensive computer workstations with sophisticated visualization software. In the VSS course students construct 3-D virtual models on average computers using inexpensive software and go beyond flat, limited 2-D images (e.g. charts, graphs) to constructing complex models.

\section{Study Context}

The VSS course is a project-based experimental undergraduate astronomy course in which students work in dyads and triads to build 3-D models of different aspects of the Solar System. The curriculum for the course is comprised of three modeling projects of increasing complexity designed to engage students in modeling various astronomical phenomena that are typically covered in a traditional lecture-based class. The projects are:

1. The Celestial Sphere (a concept first envisioned by ancient astronomers to sudy the motion of the stars and planets). In this project the students construct a 3-D static model of the Celestial Sphere. The goals for the students during this project are to construct a geocentric model of the Earth-Sun system to learn the essential astronomical terminology (e.g. right ascension, declination), to learn the causes for the seasons, and to build a conceptual base that they will use to understand future astronomy concepts. 
2. The Earth-Moon-Sun system: In this project students construct a 3-D dynamic model of the Earth-MoonSun system. The students are expected to investigate the orbital paths, periods, distances between, rotational rates, and the relationships between these bodies. The students also compare their model with the real Earth-Moon-Sun system and report on any discrepancies between the two.

3. The Solar System: This project consists of students constructing a 3-D dynamic model of the Solar System. They are expected to build a model of the Solar System that takes into account the rotational and revolutional rates of the planets, and the relative sizes and distances between the planets. In constructing their models, students grapple with the difficult concept of the vastness of scale of the solar system. Lastly, they investigate the similarities and differences between the planets' orbital motions, spins, interior structures, moon systems, and atmospheres.

The virtual solar system models are expected to address both instructor and student constructed questions relating to important astronomical phenomena. The student teams expand these questions as they construct, revise, and evaluate their models. Each team develops strategies to answer the questions, identifies resources and designs, and builds their models by dividing up the tasks inherent to the project. At the conclusion of each project, the students are given a range of opportunities (presenting their model to the class for peer review and writing a group paper describing their model) to reflect on the model construction process, and the astronomy concepts demonstrated through and embodied in their models.

Students constructed their models using a Virtual Reality Markup Language (VRML) wyswig editor, CosmoWorlds, freeing the students from concentrating on learning how to program allowing students to focus their efforts toward learning astronomy. VRML is similar to HTML in that it is the standard language used for viewing VR on the World Wide Web. VRML is platform independent, object oriented, and is easily viewed over the Web using a free plug-in and a web browser. The ease of porting students' VRML projects to the Web is a large motivating factor for the students because they are aware that their models can be viewed by their peers and critiqued by anyone who has Web access. Further, VRML easily allows students to place different "viewpoints" in their models. A viewpoint allows the students to locate a camera at important positions (i.e. standing on the Moon to observe a solar eclipse) to view different astronomical phenomenon within their model (see figure 1).

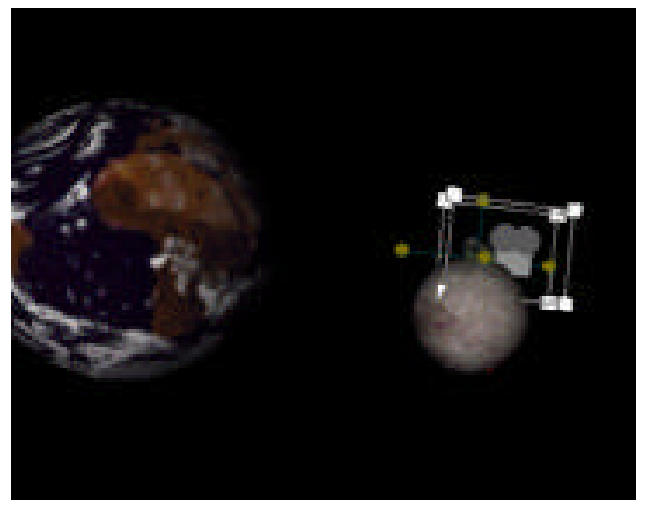

Figure 1. Screenshot of students placing a viewpoint on the Moon to observe the Earth

\section{Study Methods}

The eight undergraduate students that participated in the Summer 1998 VR section of Introduction to Astronomy were interviewed twice, once at the beginning of the course, and once immediately following the completion of the course. The interview questions were semi-structured, consisting of nine questions that covered a wide range of astronomy concepts that are typically covered in traditional Introduction to Astronomy courses. The questions were derived from the alternative conception research, and from consultation with faculty members from the Astronomy Department at Indiana University. In this manuscript, we focus on two of the interview questions that 
explore basic astronomical concepts that represent the strengths and weaknesses of eliciting conceptual change through 3-D modeling projects. The questions that are the focus of this study are as follows:

Eclipses and Phases: What are differences and similarities between a Full Moon and a Lunar Eclipse?

Reasons for the Seasons: What causes the seasons of the Earth?

The pre-interviews were videotaped and conducted during the first two days of the class to capture students' conceptual understanding prior to their constructing 3-D models. The students were asked to express their understandings verbally, and were provided with a set of spheres for manipulation and a white board for drawing to demonstrate their explanations. The interviewer asked probing questions to establish the depth of students' conceptual understanding. The post-interviews were videotaped and conducted during the last week of the course. Again, the students were asked to express their understandings either verbally, as they manipulated spheres, or by drawing on the available white board.

\section{Data Analysis}

We assessed student conceptual growth by extensive viewing of the videotapes, analysis and coding of the transcribed interviews, and scoring the student responses by a rubric. The rubric is based upon the categorization scheme used by Simpson and Marek (1988) and Muthukrishna, Carnine, Grossen and Miller (1993), and was modified slightly to reflect a hierarchical developmental sequence (see table one for eclipses and phases rubric). The rubric has five categories as follows: (0) No conception, (1) Confused, (2) Incomplete/Inaccurate Understanding, (3) Partial Understanding, and (4) Complete Understanding. Both the pre and post-interview responses were scored using a rubric adapted to the question of interest.

Both the course instructor and the researcher/interviewer scored every pre and post-interview question with the developed rubric. A correlation analysis was performed to determine the level of inter-rater reliability. The agreement between the researchers for question one was $\underline{r}_{\text {pre }}=0.94$, and $\underline{r}_{\text {post }}=0.96$, question two was $\underline{r}_{\text {pre }}=0.90$, and $\underline{\mathrm{r}}_{\text {post }}=0.96$.

\section{Results}

Students showed significant improvement in their conceptual understanding concerning the phases and eclipses of the Moon $\left(\chi^{2}(4,8)=5.85, \mathrm{p}<.01\right)$, and reasons for the seasons $\left(\chi^{2}(4,8)=5.96, \mathrm{p}<0.01\right)$. The majority of students began the VR course with an impoverished understanding of what causes lunar eclipses and the phases of the moon.

By the end of the course, all but one student arrived at a solid conceptual understanding of eclipses and phases (see figure 1). The concept of the line of nodes, in combination with the concept of the Moon's orbital tilt, emerged as invaluable conceptual tools during the modeling process. These conceptual tools facilitated the students' acquisition of a robust conceptual framework of complex astronomical phenomena. For example, in the below dialogue Sally did articulate a response when asked about the Moon's phase, but grew confused and had an alternative framework regarding the position of the Moon during her pre-interview:

Interviewer: We have the Sun, the Moon and the Earth. I want to know how they are positioned relative to each other to get a full moon.

Sally: $\quad$ I don't know. I know my eclipses.

Interviewer: Ok, so tell me about a lunar eclipse.

Sally: I believe it would be like this [places the Moon sphere between the Earth and the Sun sphere]. I don't know. Maybe behind the Earth? [Puts the Moon sphere opposite the Sun, behind the Earth sphere]

Interviewer: So sitting on the Earth, which position would the Moon get dark.

Sally I guess this one [the one with the moon behind the Earth]. This is the moon we see during the day. Puts the moon beside the Sun. This one see at night puts the moon behind the Earth

Interviewer: So where does the Moon have to be to give us a full moon. 
However, in the post interview, Sally called upon the conceptual tool of the line of nodes that her team has modeled during the second project.

Interviewer: So tell me about a lunar eclipse.

Sally: $\quad$ A lunar eclipse can only occur when the Moon is on the line of nodes.

Interviewer So what are the differences between a full moon and a lunar eclipse?

Sally: $\quad$ It is when the Moon is on the line of nodes. It has to be on the line of nodes.

Interviewer: So what does that mean?

Sally: It is imaginary, but not really. It is if everything is lined up perfectly. We get a lunar eclipse.

Interviewer: So why don't we get a lunar eclipse every month?

Sally: $\quad$ Because the Moon doesn't hit the line of nodes every month. Then you get into the differences between sidereal and synodic. For example, a sidereal time is when you see the moon against the same background stars. That also plays a point in the line of nodes, because the moon is not going to be in the spot at the same time the next day.

Three quarters of the students entered the VR course with a range of common alternative frameworks regarding the seasons as reported in previous studies (Pfundt \& Duit, 1998) including explaining the seasons as caused by the distance from the Sun and the Earth's rotation. In the post-interviews, every student identified the tilt of the Earth's axis as the primary cause of the seasons. However, only three students obtained a complete understanding (see figure 2). For example, in Jason's post-interview response he had a hybridized understanding between the classic conception that the seasons are caused by the distance the Earth is away from the Sun and the more scientifically correct reason, namely the tilt of the Earth.

Interviewer: What causes the seasons of the Earth?

Jason: What causes the seasons of the Earth? (Holding spheres). You know, here's the sun and here's the Earth, the Earth is rotated on its axis $231 / 2$ degrees, and that's what actually makes the seasons. As it rotates around the sun 365 days, the tilt causes different regions of the earth to be closer to the sun or farther from the Sun in the 365 day revolution which causes the seasons.

Interviewer: What do you mean by closer and further away?

Jason: $\quad$ Closer and further away, OK. Here's the earth and here's the Sun. (Still working with two spheres). Here is the sun at the summer solstice up here. Say here's North America.

This is the position of the Sun at the summer solstice (holding Sun up above Earth). I'm all confused now... Earth and Sun at the summer solstice, this band, what is it?

The Tropic of Cancer, and during the summer solstice it's kind of rotating like this. And the Tropic of Cancer is closest actually in distance from the Sun to the Earth, it's the closest spot.

As it comes around the earth begins spinning pretty much like that and the closest distance from the sun to the earth is the equator, right there in the middle, at the equinox with equal day and equal night. Now I'm up here at the winter solstice and the closest spot is the Tropic of Capricorn down here as the Earth rotates around.

When the other students were asked to expand on their answers, only three of the eight students were able to provide an adequate explanation of the seasons that included the importance of the angle of incidence of sunlight on the Earth's surface (see figure 3). This lack of student understanding of the role of incident solar radiation on the Earth's surface as the major factor influencing the seasons can be attributed to the limitations of the CosmoWorlds software, and the static nature of the first modeling project. During much of the first project students were 
concerned with imitation, rather than representation and explanation, thus they were not engaged in hypothesis formulation and modeling questioning that characterized the latter projects. The student groups expressed a desire to include the illumination of the Earth's surface in their models, but were unable to model this phenomenon with the software. Subsequently, a majority of students were unable to visualize how the incident light changes as the Sun's altitude shifts during the course of year, and were unable to adequately explain the causes of the seasons during the post-interview.

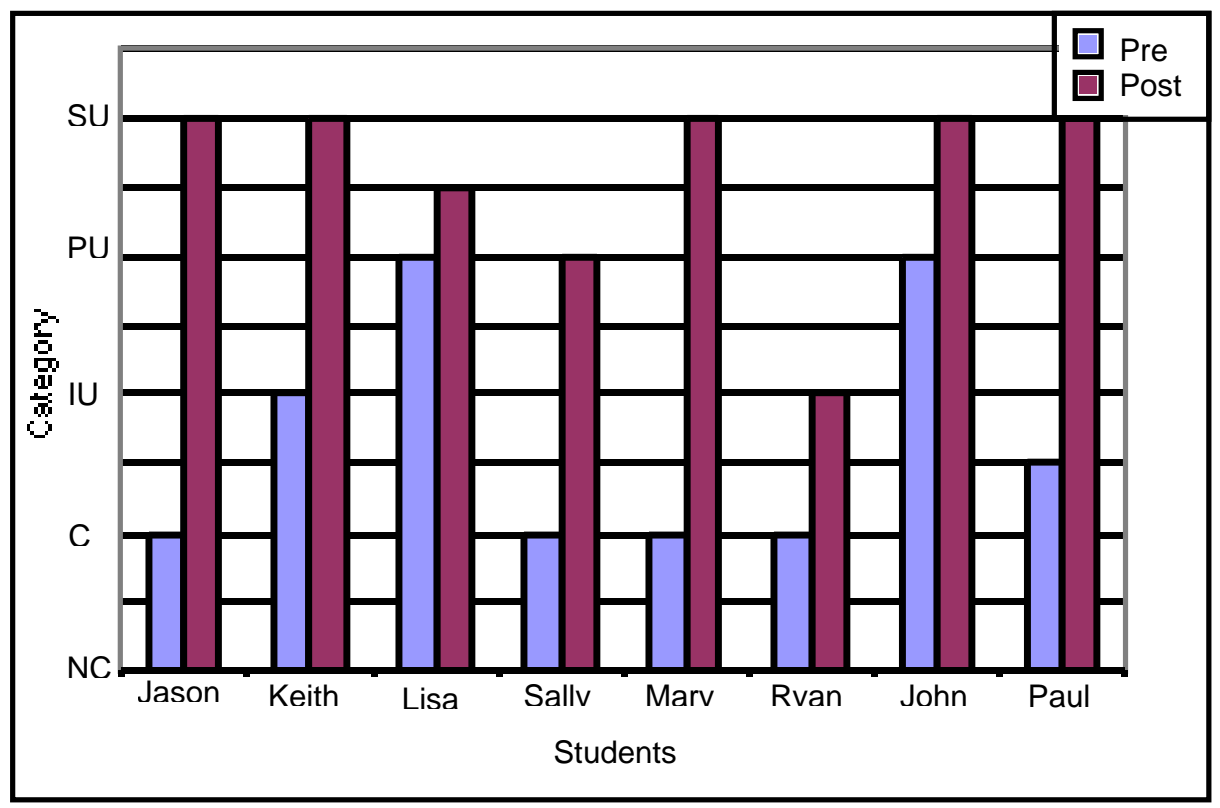

Figure 2. Student performance on the similarities and differences between a eclipses and phases of the Moon

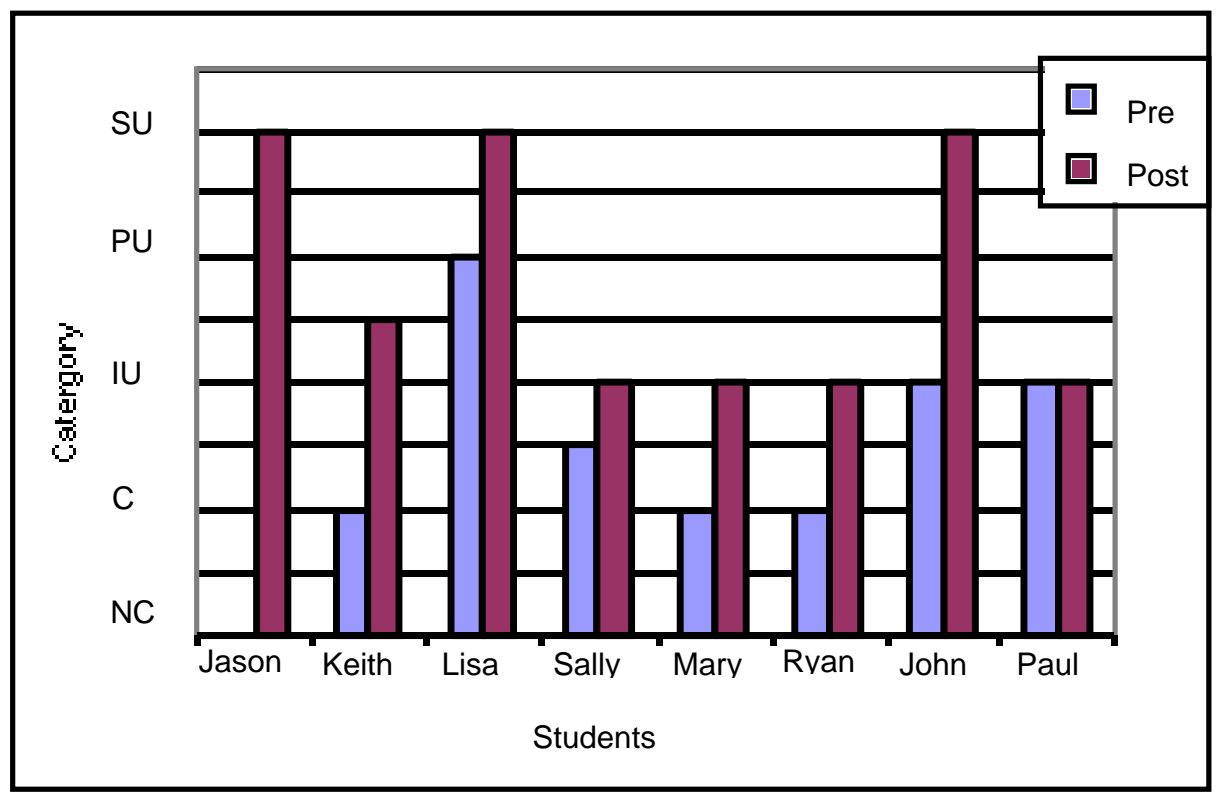

Figure 3. Student performance on the reasons for the Earth's seasons 


\section{Discussion}

From the results discussed above, it appears that all eight students that participated in VSS course experienced significant gains in their astronomy conceptual knowledge of the cause of the seasons on Earth, phases of the Moon, and eclipses. The VR modeling environment seemed to be particularly effective in supporting student learning in two fundamental ways:

1. Allowing students to construct 3-D models and view them from several different perspectives.

2. Supporting students' ability to visualize abstract concepts such as the line of nodes. The students were able to change their perspective by navigating through 3-D space and study their models from different angles.

As part of the 3-D modeling process each group engaged in the process of deciding where to set viewpoints and how to get them to represent the astronomical concept at hand. Students positioned themselves at various positions on or near the Earth to determine when and if eclipses would occur at particular locations, when the Sun would be directly overhead, and capture the moments when the Earth, Moon, and Sun were all on the line of nodes. This navigational ability not only allowed the students to place themselves in the role of the Earth, Sun, third person observer, or the Moon, but also provided them with a powerful tool to test and revise their models. For example, students could make a prediction on when an eclipse would occur and then view their model to determine the accuracy of their prediction. If an eclipse did not occur in their model the students would re-evaluate their model with the intent of determining whether their model or the assumptions they used to create their model were incorrect.

The virtual modeling environment also supported the students' ability to visualize abstract 3-D concepts. For example, in project two, the concept of the line of nodes emerged as a conceptual tool that became a fundamental component to students' understanding the differences and similarities between a full Moon and a lunar eclipse. The concept of the line of nodes is usually considered too difficult for beginning astronomy students, and frequently removed from the curriculum. However, in the act of modeling the Earth, Moon, and Sun System, every student found the line of nodes to be central to the model building process. The concept of line of nodes underwent a transformation from an abstract concept to a conceptual tool supporting student visualization and understanding of complex astronomical phenomena.

We acknowledge the limitations of the results reported in this pilot study of student learning in a VR astronomy course. The low class size of eight students may have had a significant positive effect on the overall classroom and group dynamics. Low class size facilitates greater opportunities for interaction with the instructor and potentially greater learning outcomes. During spring of 1999, the VSS course was revised using what we learned from the prototypes (Hay, et al., in press), and was offered to two sections of students with class sizes of fifteen and seventeen at Indiana University, and another VSS course was taught during the summer 1999 at the University of Georgia. We have conducted another set of pre-post interviews, with the addition of a Web-based comprehensive conceptual astronomy exam for both the VSS courses and a traditional introduction to astronomy course. Further, we are current developing a smaller module of the VSS project that integrates into the existing science curriculum of a typical middle school science classroom in which the students construct models focusing on the Earth-Moon-Sun system.

\section{Implications}

As demonstrated in the Virtual Solar System course, we now have the technological tools to assist astronomy students in visualizing abstract 3-D astronomical phenomena, which is crucial if students are to grasp the inherent 3-D nature of astronomy (Parker, \& Heywood, 1998). For example, the causes of eclipses cannot be adequately represented in a 2-D format because the Moon is not in the same orbital plane as the Earth and the Sun, but titled at an angle of five degrees relative to the ecliptic plane. It is necessary that the modeling environment reflect this inherent 3-D nature of astronomy. The incorporation of 3-D modeling activities in science courses facilitates student reflection and evaluation of their conceptual frameworks (Friedman, \& diSessa, 1999).

Our results add to the growing literature that when students are engaged in the active design and construction of models they create meaning through and from their constructions while developing scientifically sophisticated understandings of scientific phenomena (Roth, 1996, White, \& Frederiksen, 1998). As part of the model building process a "conversation" unfolds in which robust interactions occur between the student, their model and the materials of their work (Barab, et al., in press). This conversation guides the students to evaluate how they 
constructed their models_-does their model reflect the real system adequately? As a result, students move beyond simply memorizing facts, and instead, become involved in an iterative process in which their understandings inform the development of their models and the evaluation and testing of their models inform their evolving understanding (Penner, et al., 1998).

\section{References}

Atwood, R. K., \& Atwood, V. A. (1996). Pre-service elementary teachers' conceptions of the causes of the seasons. Journal of Research in Science Teaching, 33, 553-563.

Barab, S. A. Hay, K. E., Squire, K, \& Barnett, M., Schmidt, R., Karrigan, K., Ymagata-Lynch, L. \& Johnson, C. (in press). Virtual solar system project: Developing scientific understanding through model building. To appear in Journal of Science Education and Technology.

Confrey, J., \& Doerr, H. M. (1994). Student modelers. Interactive Learning Environments, 3, $199-217$.

Demastes, S. S., Good, R.G., \& Peebles, P. (1995). Students' conceptual ecologies and the process of conceptual change in evolution. Science Education, 79, 637-666.

Gilbert, J. K., \& Watts, D. M. (1983). Concepts, misconceptions and alternative conceptions: Changing perspectives in science education. Studies in Science Education, 10, 61-98.

Friedman, J., \& diSessa, A. (1999). What students should know about technology: The case of scientific visualization, 8(3), 175-197.

Lehrer, R., Horvath, J., \& Schauble, L. (1994). Developing model-based reasoning. Interactive Learning Environments, 4(3), 219-231.

Muthukrishna N., Carnine, D., Grossen, B., \& Miller S. (1993). Children's Alternative Frameworks: Should they be directly addressed in science ehducation? Journal of Research in Science Teaching, 30, 233-248.

Penner D. E., Lehrer, R., \& Schauble, L. (1998). From physical models to biomechanics: A design-based modeling approach. The Journal of the Learning Sciences, 7(3\&4), 429-449.

Pfundt, H., \& Duit, R. (1998). Students' Alternative Frameworks and Science Education. Bibliography. $5^{\text {th }}$ ed. Kiel Univ. (West Germany). Institut fuer die Paedagogik der Naturwissenschaften

Pyramid Film \& Video. (1988). A private universe. An insightful lesson on how we learn [Film]. Santa Monica, CA.

Roth, W.-M. (1996). Knowledge diffusion in a grade 4-5 classroom during a unit of civil engineering: An analysis of a classroom community in terms of its changing resources and practices. Cognition and Instruction, 14, 170-220.

Sadler, P. (1987). Misconceptions in astronomy. In J. Novak (Ed.), Proceedings of the Second International Seminar on misconceptions and educational strategies in science and mathematics, Vol III. Ithaca, NY: Cornell University

Sadler, P., Whitney, C., Shore, L., Deutsch, F. (1999). Visualization and Representation of Physical Systems: Wavemaker as an aid to conceptualizing wave phenonmenon. Journal of Science Education and Technology, 8(3), 197-211.

Simpson, W. D., \& Marek, E. A. (1988). Understandings and misconceptions of biology concepts held by students attending small high schools and students attending large high schools. Journal of Research in Science Teaching, 25, 361-374

Smith, J. P., diSessa, A. A., \& Roschelle, J. (1993). Misconceptions reconceived: A constructivist analysis of knowledge in transition. The Journal of the Learning Sciences, 3 (2), 115-163.

Stewart, J., Hafner, R. Johnson, S., \& Finkel, E. (1992). Science as model building: Computers and high-school genetics. Educational Psychologist, 27, 317-336.

Stratford, S. J., Krajcik, J., \& Soloway, E. (1998). Secondary students' dynamic modeling processes: analyzing, reasoning about, synthesizing, and testing models of stream ecosystems. Journal of Science Education and Technology, 7(3), 215-234.

Strike, K.A., \& Posner, G.J. (1992). A revisionist theory of conceptual change. In R. A. Duschl \& R. J. Hamilton (Eds.), Philosophy of Science, Cognitive Psychology, and Educational Theory and Practice (pp.147-176). New York: State University of New York Press.

Wandersee, J. H., Mintzes, J. J., \& Novak, J. D. (1990). Research on alternative conceptions in science. In D. L. Gabel (Ed.), Handbook on science teaching and Learning (pp. 177-210). New York: Macmillan Publishing Co.

White, B., \& Frederiksen, J. (1998). Inquiry, modeling, and metacognition: Making science accessible to all students. Cognition and Instruction, 16(1), 3-118. 